\title{
ERRATUM: GLOBAL STABILITY IN CHEMOSTAT-TYPE COMPETITION MODELS WITH NUTRIENT RECYCLING*
}

\author{
SHIGUI RUAN ${ }^{\dagger}$ AND XUE-ZHONG HE
}

\begin{abstract}
This note corrects some typos and errors in the paper [S. Ruan and X.-Z. He, SIAM J. Appl. Math., 58 (1998), pp. 170-192].
\end{abstract}

Key words. competition, global stability, nutrient recycling

DOI. $10.1137 / 06065876 \mathrm{X}$

The main error in the paper [1] is that conditions on the matrix $B$ should be replaced by conditions on the matrix $A B$ at four separate places.

1. On page 177 , condition (iii) in Theorem 2.8 should read

(iii) the matrix $A B$ is semipositive definite.

2. On page 182 , there were a few typos in Theorem 3.8 and an error in condition $(\mathrm{v})$. The corrected version of the theorem is as follows.

THEOREM 3.8. Assume that

(i) system (3.1) has a positive equilibrium $E^{*}=\left(S^{*}, N_{1}^{*}, N_{2}^{*}\right)$;

(ii) $D+D_{i}<m_{i}, b_{i} D_{i}<\mu_{i} p\left(S_{i}^{*}\right), i=1,2$;

(iii) $T_{f}<\infty, T_{i}^{*}=\left(1 / d_{i}^{*}\right) \int_{0}^{\infty} F(s)\left[e^{d_{i}^{*} s}-1\right] d s<\infty$ with $d_{i}^{*}:=\left(D+D_{i}\right)+$ $\sum_{j=1}^{2} \delta_{i j} m_{j}, i=1,2$;

(iv) $b_{i} D_{i}\left[\left(m_{i}+\sum_{j=1}^{2} \delta_{i j} N_{j}^{*}\right) T_{i}^{*}+m_{i} T_{f}\right] / 2<\mu_{i}, i=1,2$;

(v) The matrix $A B$ is semipositive definite, where $A=\operatorname{diag}\left(\alpha_{1}, \alpha_{2}\right)$ with $\alpha_{i}=$ $\left[\mu_{i} p\left(S_{i}^{*}\right)-b_{i} D_{i}\right] / m_{i}(i=1,2)$ and $B=\left(b_{i j}\right)_{2 \times 2}, b_{i j} \geq 0$ defined by

$$
b_{i j}= \begin{cases}\delta_{i i}-\frac{T_{f} m_{i}}{2\left[\mu_{i} p\left(S^{*}\right)-b_{i} D_{i}\right] N_{i}^{*}} \sum_{j=1}^{2} b_{j} D_{j} \delta_{j i} m_{j} & \text { if } i=j \\ \delta_{i j} & \text { if } i \neq j .\end{cases}
$$

Then $E^{*}$ is global asymptotically stable.

3. On page 186 , condition (iii) in Theorem 4.3 should read

(iii) the matrix $A B$ is semipositive definite.

4. On page 188, Theorem 4.6 should read as follows.

THEOREM 4.6. Assume that

(i) system (4.11) has a positive equilibrium $E^{*}=\left(S^{*}, N_{1}^{*}, \ldots, N_{n}^{*}\right)$;

(ii) $D+D_{i}<m_{i}, b_{i} D_{i}<\mu_{i} p\left(S_{i}^{*}\right), i=1,2, \ldots, n$;

(iii) $T_{f}<\infty, T_{i}^{*}=\left(1 / d_{i}^{*}\right) \int_{0}^{\infty} F(s)\left[e^{d_{i}^{*} s}-1\right] d s<\infty, i=1,2, \ldots, n$, where $d_{i}^{*}=\left(D+D_{i}\right)+\sum_{j=1}^{n} \delta_{i j} m_{j}$;

(iv) $b_{i} D_{i}\left[\left(m_{i}+\sum_{j=1}^{n} \delta_{i j} N_{j}^{*}\right) T_{i}^{*}+m_{i} T_{f}\right] / 2<\mu_{i}, i=1,2, \ldots, n$;

(v) the matrix $A B$ is semipositive definite, where $A=\operatorname{diag}\left(\alpha_{i}\right)_{n \times n}$ with $\alpha_{i}=$

${ }^{*}$ Received by the editors May 3, 2006; accepted for publication (in revised form) August 7, 2006; published electronically October 24, 2006.

http://www.siam.org/journals/siap/66-6/65876.html

${ }^{\dagger}$ Department of Mathematics, University of Miami, P.O. Box 249085, Coral Gables, FL 331244250 (ruan@math.miami.edu).

${ }^{\ddagger}$ School of Finance and Economics, University of Technology, P.O. Box 123, Broadway NSW 2007, Sydney, Australia (tony.he1@uts.edu.au). 
$\left[\mu_{i} p\left(S_{i}^{*}\right)-b_{i} D_{i}\right] / m_{i}(i=1, \ldots, n)$ and $B=\left(b_{i j}\right)_{n \times n}$ with $b_{i j} \geq 0$ defined as follows:

$$
b_{i j}= \begin{cases}\delta_{i i}-\frac{T_{f} m_{i}}{2\left[\mu_{i} p\left(S^{*}\right)-b_{i} D_{i}\right] N_{i}^{*}} \sum_{j=1}^{n} b_{j} D_{j} \delta_{j i} m_{j} & \text { if } i=j, \\ \delta_{i j} & \text { if } i \neq j .\end{cases}
$$

Then $E^{*}$ is globally asymptotically stable.

Acknowledgment. The authors would like to thank Karl Hadeler and Julia Hesseler for noticing the errors.

\section{REFERENCE}

[1] S. Ruan And X.-Z. He, Global stability in chemostat-type competition models with nutrient recycling, SIAM J. Appl. Math., 58 (1998), pp. 170-192. 